\title{
Complete Superior and Inferior Vena Cava Obstruction Associated with Systemic-to- Pulmonary Venous Shunts in a Young Female with Heterozygous Prothrombin G20210A Gene Mutation
}

\author{
Ashraf Omer Elamin Ahmed ${ }^{a} \quad$ Khaled A. Elfert $^{a} \quad$ Ahmed E. Mahfouz $^{\text {b }}$ \\ Fahmi S. Othman ${ }^{c}$ Lenah A. Elgassim ${ }^{d}$ Mohamed A. Yassin ${ }^{e}$ \\ aDepartment of Medicine, Hamad Medical Corporation, Doha, Qatar; \\ bepartment of Diagnostic Radiology, Hamad Medical Corporation, Doha, Qatar; \\ 'Department of Cardiology, Heart Hospital, Hamad Medical Corporation, Doha, Qatar; \\ dDepartment of Clinical Pharmacy, Heart Hospital, Hamad Medical Corporation,

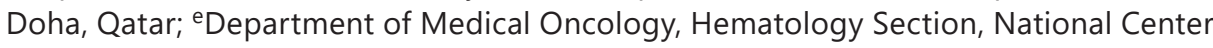 \\ for Cancer Care and Research, Hamad Medical Corporation, Doha, Qatar
}

\section{Keywords}

G20210A mutation · Inferior vena cava thrombosis · Prothrombin gene mutation ·

Right-to-left shunt - Superior vena cava thrombosis . Systemic-to-pulmonary venous shunt .

Thrombophilia

\begin{abstract}
Complete superior vena cava (SVC) and inferior vena cava (IVC) obstruction is not uncommon and most commonly associated with malignancy. The risk increases in patients with central lines and hypercoagulable states such as with malignancy, thrombophilia, or use of oral contraceptive pills. According to our knowledge, complete SVC and IVC obstruction associated with systemic-to-pulmonary venous shunts in patients with prothrombin G20210A gene mutation has not been reported in the literature. Here we report the case of a 34-year-old female with complete SVC and IVC obstruction presenting with oxygen desaturation and shortness of breath due to systemic-to-pulmonary venous shunts. The unusual collateral pathway was secondary to SVC obstruction. The patient was managed conservatively, and she remained stable.


Ahmed et al.: Complete SVC and IVC Obstruction with Systemic-to-Pulmonary Venous Shunts

\section{Introduction}

Superior vena cava (SVC) and inferior vena cava (IVC) syndromes result from complete occlusion of the caval veins, which are considered the great vessels for venous return to the heart. Their presentation varies and depends on the degree and duration of the obstruction and the formation of collateral vessels, as it is sometimes considered a medical emergency if the blockage is acute and needs immediate intervention [1]. The most common symptoms of SVC obstruction are headache, shortness of breath (SOB), facial plethora, upper limb edema, and distended neck and upper chest veins [2]. IVC obstruction commonly presents with lower limb edema, tachycardia, and supine hypotensive syndrome [3].

Prothrombin G20210A gene mutation is the second most common inherited thrombophilia after factor $\mathrm{V}$ Leiden mutation. It is most common among Caucasian, and infrequent among Asian and African populations [4]. It increases the risk of venous thromboembolism (VTE) 3- to 5-fold compared to the normal population. It is suspected in patients with recurrent VTE, VTE with a positive family history of recurrent VTE, or VTE with a known family history of prothrombin gene mutation [5]. Historically, the classic presentations with prothrombin G20210A gene mutation are deep venous thrombosis (DVT), pulmonary embolism (PE), portal venous thrombosis (PVT), hepatic and mesenteric venous thrombosis, and BuddChiari syndrome (BCS) [5]. Cases of cerebral vein thrombosis and retinal vein thrombosis [5] are reported as well.

Here, we report a case of SVC and IVC obstruction associated with systemic-to-pulmonary venous shunts (SPVS) in a patient who has heterozygous prothrombin G20210A gene mutation.

\section{Case Report}

We present the case of a 34-year-old Pakistani woman known to have heterozygous prothrombin G20210A gene mutation with a history of recurrent PE, cerebral vein thrombosis (superior sagittal and transverse sinuses), and left leg DVT. She was on warfarin, but she was not compliant with medical treatment. She presented to the hospital with SOB and desaturation. On examination, she was morbidly obese; her BMI was $52.5 \mathrm{~kg} / \mathrm{m}^{2}$. She had a respiratory rate of 20 breaths/min, a heart rate of 82 beats/min, and a blood pressure of $104 / 60 \mathrm{~mm} \mathrm{Hg}$. Her oxygen saturation was $80 \%$ on room air, improved with $4 \mathrm{~L}$ of oxygen to $92 \%$. Her face looked plethoric, but she had no central cyanosis.

Chest examination revealed normal vesicular breathing with no added sounds. Cardiac examination showed normal heart sounds with no murmurs. There were dilated veins in her chest anteriorly. She had dilated superficial abdominal veins. Her lower limbs showed varicose veins, with mild bilateral edema. For her blood test results, see the online supplementary Labs file (for all online suppl. material, see www.karger.com/doi/10.1159/000507283).

Chest X-ray showed a normal lung parenchyma. The patient was started on enoxaparin; however, her saturation was still around $80-85 \%$ room air. Because of the high likelihood of PE, CT angiography of the chest was done. It showed complete SVC occlusion with extensive collaterals. The pulmonary arteries were not opacified because of the SVC occlusion. The collaterals were seen connecting the tributaries of the SVC to the pulmonary veins (Fig. 1). Her echocardiography revealed an ejection fraction of $30 \%$. A repeated echocardiogram with bubble study showed that the agitated saline injection was filling the left side of the heart completely, with no filling of the right side (online suppl. Video).

Afterward, the case was discussed with the interventional radiology team, and a procedure was done to insert a coil in the shunt connecting the SVC tributaries to the pulmonary veins. 
Ahmed et al.: Complete SVC and IVC Obstruction with Systemic-to-Pulmonary Venous Shunts
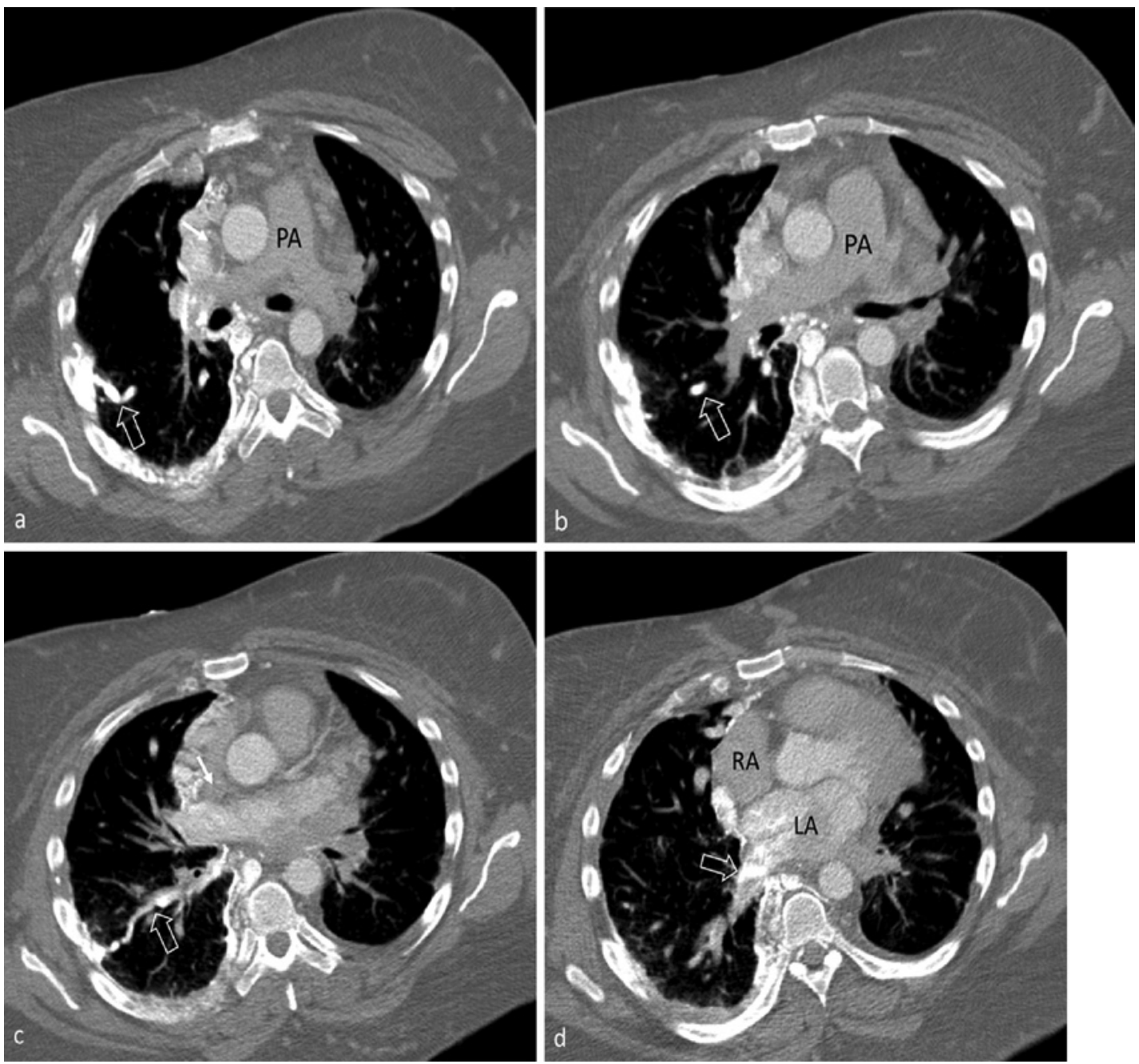

Fig. 1. a-d Contrast-enhanced CT showing thrombosis of the superior vena cava (solid arrows) venous collaterals of the chest wall and mediastinum and systemic-to-pulmonary venous shunts to the left atrium (LA), while the right atrium (RA), right ventricle, and pulmonary artery (PA) show a less dense contrast agent content during the first pass of the intravenously injected contrast agent.

The patient was discharged on rivaroxaban because of poor compliance with warfarin. The patient was reassessed 6 months after the procedure, and there was no improvement in oxygen saturation. After 1 year, she was diagnosed with PVT while she was on rivaroxaban, so it was decided to shift her back to warfarin. Because of the persistent hypoxemia despite the coiling of the shunt and the optimal treatment of her heart failure, her condition was re-discussed in a multidisciplinary team meeting. We planned to insert a stent into the SVC to relieve the obstruction. The rationale was that stenting the SVC would result in collapse of the SPVS. An IVC cavogram (Fig. 2, 3) was done to assess the port for extracorporeal membrane oxygenation if needed. It showed complete obstruction of the infrahepatic part of the IVC with extensive collateral vessels. The procedure was deferred because of the presence of complete IVC obstruction. The patient was discharged on home oxygen with requirements of 1-2 L/ min and $13 \mathrm{mg}$ of warfarin with close follow-up. 
Fig. 2. Superior vena cavogram demonstrating complete occlusion of the superior vena cava.

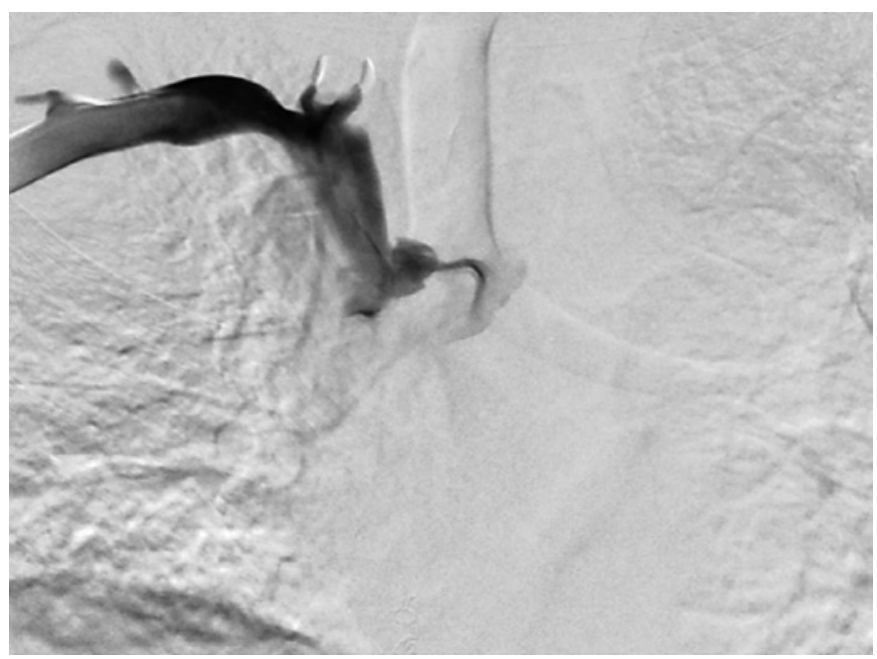

Fig. 3. Inferior vena cavogram demonstrating occlusion of the infrahepatic part of the inferior vena cava replaced by venous collaterals.

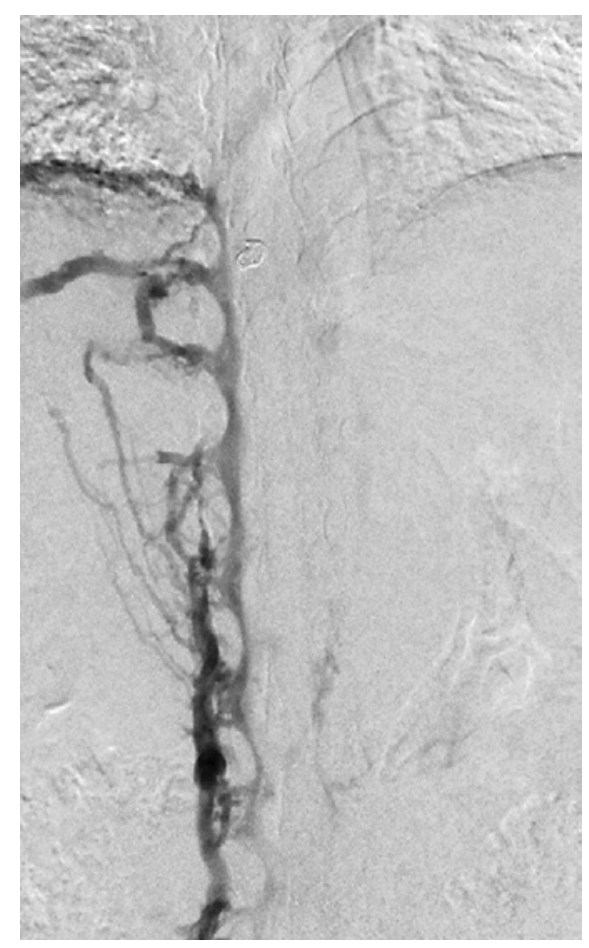

\section{Discussion}

Prothrombin G20210A gene mutation is proven to increase the risk of VTE 3- to 5-fold. It is associated with DVT, PE, PVT, BCS, mesenteric ischemia, retinal vein thrombosis, cerebral vein thrombosis, and recurrent early pregnancy loss, as well as with arterial thrombosis (stroke and coronary artery disease) [5-7]. However, complete SVC and IVC obstruction has not been reported in association with prothrombin G20210A gene mutation. Generally, malignancies are known causes of caval obstruction, either by a compression effect or an increased coagulability status of the patient [8]. Central lines are also risk factors even in nonthrombophilic patients [9], as well as hormonal medications, which can amplify the risk of VTE in thrombophilia patients compared to normal patients [10]. An unusual case of Behçet 
Fig. 4. Color Doppler ultrasonogram of the liver showing patency of the hepatic veins and the suprahepatic part of the inferior vena cava.

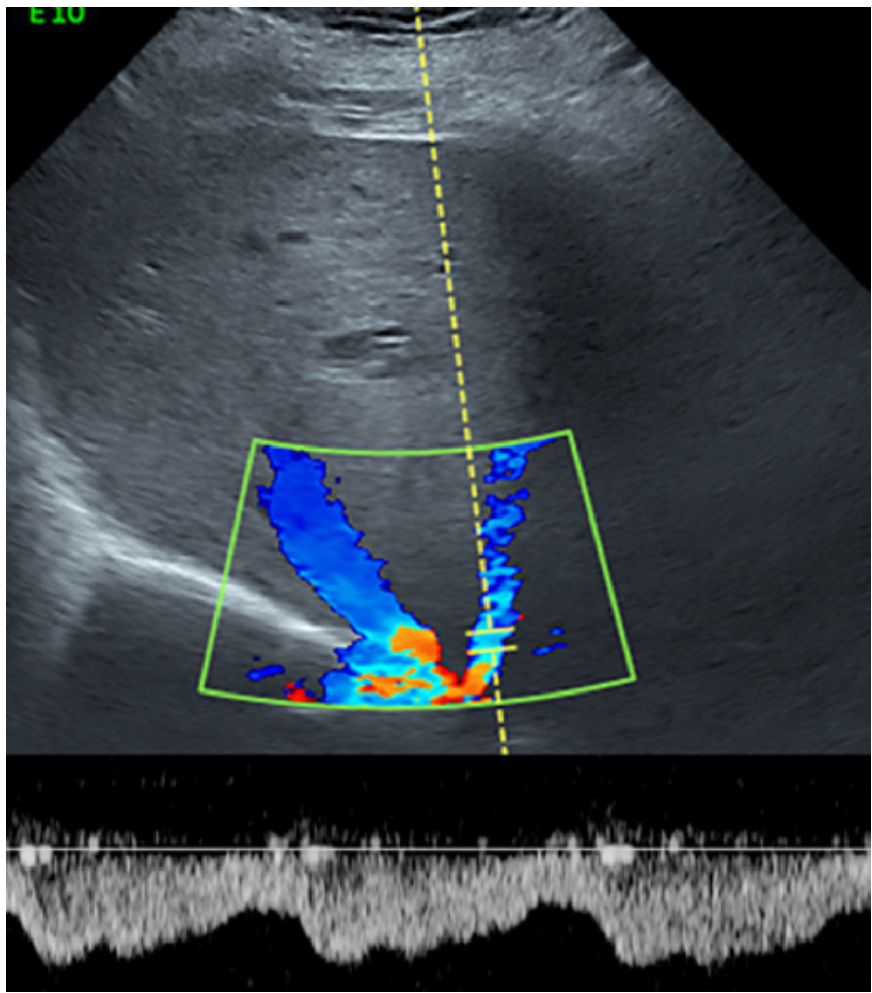

syndrome with complete caval vein obstruction has been reported [11]. In patients with chronic occlusion, collateral pathways will develop to maintain venous return to the heart. In reference to our patient, she presented with SOB and desaturation because of well-established extensive collateral vessels and shunts, as demonstrated.

SVC obstruction leads to the development of multiple venous collaterals including azygoshemiazygos, internal and external mammary, lateral thoracic, and vertebral [12]. Another rare collateral pathway that is described in case reports is the SPVS [13-15]. Upon review of the literature, we found 2 cases of SVC/IVC obstruction with SPVS [16, 17], but in those cases, the patient had also BCS, while our patient had obstruction of the infrahepatic part of the IVC only (Fig. 3, 4). Other features that were present in the 2 cases and not in our case were that the thrombosis was attributed to antiphospholipid syndrome, the IVC occlusion was subtotal, and the patient underwent successful dilatation and stenting of the SVC and IVC, which resulted in improvement of hypoxemia. Dilatation and stenting were discussed in our case, but it was decided in the multidisciplinary meeting that the procedure was technically difficult because of the complete thrombosis of the SVC and IVC.

In patients with prothrombin G20210A gene mutation, treatment is only required when complications related to thrombophilia such as VTE occur. The current VTE guidelines prefer direct oral anticoagulants (DOAC) over vitamin K antagonists for VTE. However, there is no mention of any special recommendations or exclusions for thrombophilic patients regarding DOAC usage [18]. In fact, clinicians who consider DOAC as an option rely on post hoc analysis of landmark trials or some case reports rather than well-conducted randomized trials [19, 20]. Our patient was shifted from warfarin to rivaroxaban as the INR while being on warfarin was occasionally subtherapeutic because the patient was not monitoring her INR on a regular basis and she was not compliant. Thus, she was counseled about the advantages and risks of other anticoagulants, which are not well studied in patients with thrombophilia. It was 
decided to shift her back to warfarin because of the development of a new thrombotic event while she was on rivaroxaban.

This case report is limited by the fact that heart MRI was planned to quantify the shunt and to exclude other causes of the low ejection fraction, but the patient was no able to complete the study. The low ejection fraction was attributed to the high cardiac output state secondary to the large right-to-left shunt [12].

\section{Conclusions}

There are several aspects that make this case interesting to report. First, we could not find any cases in the literature with SVC/IVC obstruction-related SPVS due to prothrombin G20210A gene mutation. Furthermore, inherited thrombophilia such as prothrombin G20210A gene mutation is a rare cause of SVC occlusion-related SPVS, and coiling was not successful as the collateral pathway was a network of veins rather than a single connection. This case also highlights the challenge of managing such cases and the scarcity of evidence for the use of anticoagulants other than warfarin in those cases.

\section{Acknowledgements}

We acknowledge the internal residency program for scientific support, as well as the Qatar National Library for funding.

\section{Statement of Ethics}

The patient gave his written informed consent to publish his case (including publication of the images).

\section{Disclosure Statement}

All authors have no conflict of interest.

\section{Funding Resources}

This case report was funded by the Qatar National Library.

\section{Author Contributions}

A.O.E. Ahmed and K.A. Elfert: manuscript writing and literature review; A.E. Mahfouz: diagnostic image interpretation and reporting; F.S. Othman: echocardiographic image interpretation and reporting; L.A. Elgassim: literature review; M.A. Yassin: team supervisor and mentor, as well as manuscript review. 
Ahmed et al:: Complete SVC and IVC Obstruction with Systemic-to-Pulmonary Venous Shunts

\section{References}

1 Cohen R, Mena D, Carbajal-Mendoza R, Matos N, Karki N. Superior vena cava syndrome: a medical emergency? Int J Angiol. 2008;17(1):43-6.

2 Wilson L, Detterbeck F, Yahalom J. Clinical practice. Superior vena cava syndrome with malignant causes. N Engl J Med. 2007;356(18):1862-9.

3 Parikh R, Beedkar A. Inferior vena cava: chronic total occlusion. Mayo Clin Proc. 2018;93(4):548.

4 Rosendaal FR, Doggen CJ, Zivelin A, Arruda VR, Aiach M, Siscovick DS, et al. Geographic distribution of the 20210 G to A prothrombin variant. Thromb Haemost. 1998;79(4):706-8.

5 Kujovich JL. Prothrombin-related thrombophilia. In: Adam MP, Ardinger HH, Pagon RA, Wallace SE, Bean LJH, Stephens K, et al., editors. GeneReviews ${ }^{\circledR}$ [Internet]. Seattle (WA): University of Washington, Seattle; 19932020.

6 Sarecka-Hujar B, Kopyta I, Skrzypek M, Sordyl J. Association between the 20210G>A prothrombin gene polymorphism and arterial ischemic stroke in children and young adults - two meta-analyses of 3,586 cases and 6,440 control subjects in total. Pediatr Neurol. 2017;69:93-101.

7 Pickering W, Marriott K, Regan L. G20210A prothrombin gene mutation: prevalence in a recurrent miscarriage population. Clin Appl Thromb Hemost. 2001 Jan;7(1):25-8.

8 Kogoj P, Zbacnik R, Dolenc-Stražar Z, Klokocovnik T, Cvijic M. Superior and inferior vena cava obstruction: an unusual presentation of a rare primary cardiac tumour. Eur Heart J. 2018;39(27):2604.

9 Shefler A, Gillis J, Lam A, O'Connell AJ, Schell D, Lammi A. Inferior vena cava thrombosis as a complication of femoral vein catheterisation. Arch Dis Child. 1995;72(4):343-5.

10 Dayan N, Holcroft C, Tagalakis V. The risk of venous thrombosis, including cerebral vein thrombosis, among women with thrombophilia and oral contraceptive use: a meta-analysis. Clin Appl Thromb Hemost. 2008 Nov; 17(6):E141-52.

11 Yoo W, Moon J, Kim S, Kim W, Min J, Park S, et al. A case of Behçet's disease with superior and inferior vena caval occlusion. Korean J Intern Med. 1998 Jul;13(2):136-9.

12 Kapur S, Paik E, Rezaei A, Vu D. Where there is blood, there is a way: unusual collateral vessels in superior and inferior vena cava obstruction. Radiographics. 2010 Jan;30(1):67-8.

13 Cihangiroglu M, MacEneaney P, MacMahon H, Dachman A. Systemic to pulmonary venous shunt in superior vena cava occlusion. J Thorac Imaging. 2000;15(3):208-10.

14 Juan Y, Saboo S, Anand V, Chatzizisis Y, Lin Y, Steigner M. Superior vena cava syndrome associated with rightto-left shunt through systemic-to-pulmonary venous collaterals. Korean J Radiol. 2014;15(2):185-7.

15 Grayet D, Ghaye B, Szapiro D, Dondelinger R. Systemic-to-pulmonary venous shunt in superior vena cava obstruction revealed on dynamic helical CT. AJR Am J Roentgenol. 2001;176(1):211-3.

16 Bin Saeedan M, Alrujaib M, Fathala AL. Hypoxemia and right-to-left-shunt in patient with antiphospholipid syndrome: a case report with multimodality imaging findings and literature review. Case Rep Radiol. 2016; 2016:2092084.

17 Bunge J, Wiersema U, Moelker A, Van Bommel J, Tjwa E. Refractory hypoxemia in a 23-year-old patient with Budd-Chiari syndrome. Chest. 2014;146(5):e149-52.

18 Skelley JW, White CW, Thomason AR. The use of direct oral anticoagulants in inherited thrombophilia. J Thromb Thrombolysis. 2017;43(1):24-30.

19 Goldhaber S, Eriksson H, Kakkar A, Schellong S, Feuring M, Fraessdorf M, et al. Efficacy of dabigatran versus warfarin in patients with acute venous thromboembolism in the presence of thrombophilia: findings from RE-COVER ${ }^{\circledR}$, RE-COVERTM II, and RE-MEDYTM. Vasc Med. 2016;21(6):506-14.

20 Undas A, Goralczyk T. Non-vitamin K antagonist oral anticoagulants in patients with severe inherited thrombophilia: a series of 33 patients. Blood Coagul Fibrinolysis. 2017;28(6):438-42. 